\title{
Research on Intelligent Lighting System of LED Based on Wireless Sensor Network Technology
}

\author{
Zhiyuan Weng, Jie Fang, Min Kong, Ying Cheng \\ College of Mechanical and Electrical Engineering \\ West Anhui University \\ Lu-An, 237012, China
}

\begin{abstract}
With the development of mobile Internet and semiconductor technology, people have the increasingly strong demand for the home life of the network, intelligence and energy-saving. The household appliances, lighting, security alarm integrated into the control platform through a wireless network, realizing the intelligent control and management. Aiming at the defects of the current traditional lighting system about manual operation, complex wiring and so on which can't meet the requirements of the environmental sampling, linear dimming, the design of lighting control system of LED based on ZigBee wireless sensor network technology was presented. CC2530 chip was chosen and Mesh topology structure of ZigBee 2007/PRO wireless sensor network was used by the system to acquire real- time environment parameters in lighting scene, such as illumination, moving target, temperature, LED state and so on. With the change of environmental parameters and the preset lighting mode, the system can automatically make quick response and realize PWM linear non-polar dimming. The LED lighting control system was real-timely monitored and managed by the PC host computer based on the platform to overcome the defects of traditional lighting inefficient management and staff wastage. The results indicate that the system achieves the design goal of the realtime acquisition of environment parameters by sensor nodes, precise control by ZigBee remote control and centralized management by PC and the experimental data is ideal.
\end{abstract}

Keywords- intelligent lighting control; wireless sensor network technology; LED

\section{INTRODUCTION}

Accompanied by the rapid development of mobile internet, semiconductor technology and the raising of quality of modern people's life, the demand of home life with wireless, networked, intelligent, energy-saving requirement is more and more intense. People tend to make the household appliances, lighting, security alarm, home theater and other modules into the control platform to realize intelligent control and intelligent management. Intelligent lighting control system is an essential subsystem of home automation. It is an important part of building control system. In the traditional sense of people, lighting system only to lighting for the purpose, but with the rapid development of science and technology at the same time, people for home lighting control system's function demand is also growing, including site acquisition environment, body sensors, intelligent dimming color temperature, scene settings, wireless remote control, manmachine interface and so on. At present, complicated wiring, expansion of poor, artificial management, single function, serious energy and many defects, the traditional lighting control system has been unable to meet modern people's family life and quality requirements. Under this background, the intelligent lighting control system came into being. The rapid development of LED semiconductor energy-saving light source and the wireless sensor network, to the lighting control system of "network, intelligent, energy-saving" homeland concept brings a broad space for development and application prospect. ZigBee wireless sensor network technology based on positive EE802.15.4 protocol standard, with automatic network, short distance, low rate, low cost, low power consumption, and many other advantages, in the industrial automation control, intelligent building, consumer electronics and automatic meter reading and other fields have a wide application prospect. At the same time, the ZigBee Alliance for wireless sensor networks in the intelligent lighting control field defines a set of very detailed protocol standard, based on the ZigBee wireless sensor network LED intelligent lighting system is advantageous for the expansion and promotion, reduce the cost of system integration. This paper mainly aims at the key technology of intelligent LED lighting research, including constant current drive, PWM dimming, RF interference, multitask scheduling, dynamic routing algorithm and energy balance to give an improvement intelligent lighting solutions.

\section{DESIGN REQUIREMENTS OF INTELLIGENT LIGHTING SYSTEM OF LED}

\section{A. Hardware Design Requirements}

Considering ZigBee alliance specifications for the design of intelligent lighting system switch module, optical module, sensor module, RF radio frequency module and PCB antenna to ensure that between different vendors function modules can be replaced, system is fully compatible, good expansibility and can reduce LED intelligent lighting system integration difficulty. We use the patch design to reduce the drive PCB board size, and LED lamps set as one, to meet the user that is inserted, that is, for the use, convenient installation, and practical beauty. To give full play to the advantages of energy saving LED light dimming, color temperature, drive is crucial, precise 
control of current pulse frequency and duty ratio PWM electrode less dimming to reasonable lighting. On the one hand, it is required that the LED lighting lamp can save energy and save energy, reduce power consumption; on the other hand, the ZigBee module is required to reduce power consumption, extend battery power supply time, and extend the life of wireless sensor networks. Lighting environment field inevitably will not be outside interference, requirements of ZigBee module signal to noise than the outstanding anti-interference ability. It also requires wireless transmitting and receiving module satisfies the communication distance indoor wireless sensor network conditions to guarantee the stability of the communication.

\section{B. Software Design Requirements}

The software of the ZigBee module follows the ZigBee protocol specification and the modular design, with good system compatibility. Data format is the same and can improve the transmission quality and stability to reduce the delay. ZigBee wireless sensor network to realize automatic network, network flexibility, have the ability to repair itself; users can arbitrarily add, delete the ZigBee module. All modules can be set as coordinator, router or end device; module layout is reasonable, to ensure network covering a wide area, no communication blind area. Using the CC2530 chip clock management unit, the LED lamps in the LED intelligent lighting system are implemented to carry out the packet synchronization control and the time delay control. Router according to the routing query, and route maintenance command to dynamically maintain routing tables, between a source node and a target node data transmission line not only, have rerouting function to improve the reliability of communication and the ability to adapt to the environment.

\section{HARDWARE DESIGN OF INTELLIGENT LIGHTING SYSTEM OF LED}

\section{CC2530 System}

CC2530 chip selection has high performance, low power consumption and enhanced version of 8051cpu core, fully compatible with the standard 8051 instruction set, instruction cycle are single clock, eliminating the waste of bus mode, program execution speed by leaps and bounds. It also support flash hash system can be programming, 8KB RAM with data retention capacity; internal structure optimization, an increase of the second data refers to the total, extended to 18 interrupt sources, four interrupt priority. CODE, DATA, XDATA, SFR four independent storage spaces are used to store programs and data. The code $64 \mathrm{~KB}$ read-only storage space, limited to the stored program; data for $256 \mathrm{~b}$ read/write data storage space, the sheet instruction cycle access; XDATA 64KB read/write data storage space and 4-5 instruction cycle access; SFR $128 \mathrm{~B}$ read/write register storage space, the sheets of the instruction cycle access. CC2530 chip integrated support 2.4 GHz IEEE 802.15.4 Wireless transceiver, programmable output power as high as $4.5 \mathrm{dBm}$, have excellent sensitivity and strong anti-interference to link quality. The RF kernel directly controls the analog wireless module, and provides the communication interface between MCU and wireless devices, which can realize many functions such as command, read state, automatic operation and radio event sequencing.

\section{ZigBee Nodes}

In the ZigBee wireless sensor network node hardware platform and CC2530 peripheral circuit mainly includes crystal oscillation circuit, RF circuit, power supply circuit, filter circuit, sensor circuit, LCD display circuit, RS232 communication circuit and other auxiliary circuits. The crystal oscillator circuit consists of two internal RC oscillator, slightly lower precision, low power consumption, quick start; two external quartz crystal oscillator, high precision, large power consumption, slow start. 12 bit ADC for digital to analog conversion environmental parameter information collected by the sensor module; RF radio frequency to realize the input interface of a ZigBee wireless data transceiver power supply circuit selects the modulation voltage regulator 3.3V power supply, precision reference current bias resistor r301 precision control in less than $0.5 \%$ to crystal oscillation circuit provides the optimum operating current. The filter circuit is configured to work with a capacitor, which works with the power supply module to improve the stability and precision of the internal voltage of CC2530. Crystal oscillator circuits configuration two resonant capacitors, a chip CC2530 to provide accurate internal system clock and a clock to register implementation.

\section{E. LED Nodes}

LED light source luminous intensity depends on the LED light source on the forward current VF. When VF generals had led to the LED chip junction temperature significantly increased and luminous flux greatly attenuated and LED life will significantly shorten when w a teenager in LED luminous intensity, affect the quality of the lighting. Therefore, the best choice of LED source driver is the constant current power supply technology, in order to ensure the safety and efficiency of high power LED light source, to achieve the most ideal luminous intensity and lighting comfort. Pulse constant current source drive is a constant current driver IC in a new technique, the frequency of the pulse current and duty ratio to achieve precise control led forward current VF, constant current fully controlled. Because of the use of PWM pulse power supply, LED is in the intermittent working state, which can achieve the purpose of constant current drive, and greatly extend the LED life. PT4115 pulse constant current driver dimming typical circuit is shown in Figure 1.

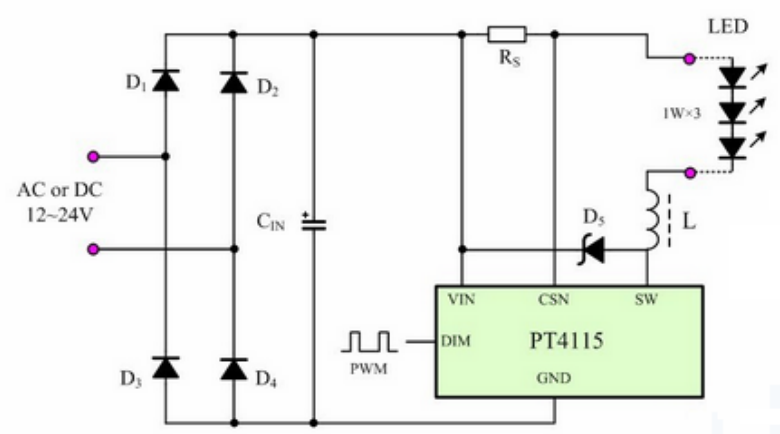

Figure 1 PT4115 pulse constant typical circuit 


\section{SOFTWARE DESIGN OF INTELLIGENT LIGHTING SYSTEM OF LED}

\section{F. Controlling Software Design of Lower Machine}

Lower machine control software includes 3 parts: the coordinator node routing node and terminal node. Coordinator is responsible for channel selection and network routing identifier maintenance boot device, and terminal and routing node collected environment information and user preset parameters by comparison, analysis, and judgment of action; routing and terminal node access success after the real-time acquisition of LED lighting control field environmental information and the working condition of LED, and the wireless coordinator transmitted back to the controller of the LED control system, the coordinator node through the RS232 serial port every $1 \mathrm{~s}$ lighting will be on-site environmental parameters and the working condition of LED PC uploaded to the PC and display, and the PC server the user control and other commands forwarded to the terminal node routing in wireless sensor networks. After the device node is activated, the device type is first judged as a full function device FFD node, and the other ZigBee network is not associated with other networks. Then FFD node by invoking the MAC layer of wireless channel scan primitives MLME-SCAN for active scanning, and set a specific time interval periodically transmits beacon request command beacon request command, set the scan period TScan-Duration. If scanning period is still not received beacon frame, then determine the FFD node within a communication area of ZigBee network, custom to the coordinator node to establish its own wireless network and continued to the ZigBee network broadcasts a beacon frame. The scanning channel includes two parts, active scanning and energy scanning. The purpose of the energy scan is to sort the scanned channels, select the good channel in the preset energy level, and discard the bad channel beyond the limit of the energy level. Then the coordinator and active scanning, active scanning is the Dayton radio beacon continuation in step 1, search the node within a communication area of a best, relatively quiet channel, the best is not associated with any other ZigBee network to avoid congestion caused by communication. After scanning the channel, the coordinator finds the best channel to meet the energy requirements.

\section{G. Monitoring Software Design of Host Machine}

Lighting system by button interface set manual mode and automatic control mode, PC machine to receive realtime lighting to the scene of the environment parameters and led working state, the moving target light according to the three parameters of the temperature in the dual form of curves and graphs display. LED state abnormal alarm to the chart PC host computer is also integrated database, the important data is automatically saved, and the user can call and view historical data and print. After the completion of the initialization of the ZigBee network, the coordinator node becomes the first device node of the new network. If the beacon frame is not received, the new node will be re initiated at a specified time interval until the network is found. When looking for the best parent node, the new node sends an association request command Request
Command Associate to the parent node immediately. The parent node then according to your own level of energy, storage space and network address factors such as the judge of the new node network request. If sufficient resources of the parent node, the parent node to the new node distribution only 16 bit network address, and will contain association network information association response to command associate response command is sent back to the new node. So far, the new node is successfully added to the ZigBee network of the parent node. If the father node resource is insufficient, new node will send connection network request command until the net success.

\section{COMMISSIONING OF INTELLIGENT LIGHTING SYSTEM OF LED}

\section{H. Self-controlled Mode Test}

First, the user click the "self-controlled" button in the interface or with ZigbeeRF4CE remote "set" key to switch to automatic control mode. The sensor module will be lighting the scene real-time acquisition of environmental parameters (light intensity, moving target and temperature) and led work state data back to the coordinator; coordinator of environmental parameters of logic judgment and intelligent analysis to make intelligent judgments and issued LED control PWM (pulse width modulation optical command; PC to a graph or diagram form of real-time display sensor module to collect environmental parameters (light intensity, moving target and temperature), LED work state and abnormal alarm information. The ZigBee node layout real LED intelligent lighting system. Respectively, set the gateway coordinator node, node 1, guest room restaurant, video room 2 node 3 and node master bedroom. Experimental results of the LED intelligent lighting system are shown in Figure 5-12, the temperature of the 4 rooms is $23.5^{\circ} \mathrm{C}, 22.2^{\circ} \mathrm{C}, 2001 \mathrm{x}$, 196bc, 2201x, 2011x,0, 3, 1 and 1, respectively, the abnormal alarm bar shows that all of the LED is working correctly, without Alarm. The reference value of the natural light illumination of LED intelligent lighting system is $210 \mathrm{k}$.

\section{Manual-controlled Mode Test}

Wall embedded switch, SgbeeRF4CE remote control and PC host computer for LED manual control, with selfmemory. First, the user in the PC interface choice in "manual mode" button or by ZigbeeRF4CE remote "set" key to switch to "manual mode", and "control" the only difference lies in the coordinator does not do any of the environmental parameters comprehensive analysis and intelligent judgment, only for as the transfer station of the transmission of the data and the control commands, the wall block switch ZigbeeRF4CE remote controller and PC lamp control button a LED control commands transmitted to all LED lamps node. PC host computer monitoring interface of the "LED lamp control" and "abnormal alarm" can be manually controlled to specify the room LED lights out and alarm. Less led 256 linear PWM has adjusting light and hotkey dimming experiment results which is convenient for the user to LED lighting system of arbitrary illumination is light and common shortcut file dimming. 
Warm color temperature adjusts color temperature LED to facilitate users to LED lighting system.

\section{J. Test Result}

After testing, the design based on ZigBee wireless sensor network has a lighting control system normal and stable operation. It has a manual control mode and an automatic control mode which can be selected. The realization of the LED according to the change of environmental parameters automatically 256 linear PWM adjusting light, color temperature, packet control, scene mode complex functions. Balance of single node module data transmitting and receiving antennas by inverted Ftype PCB antenna, and circuit board of various data multi hop transmission to meet the requirements of the receiving distance, in indoor space obstacle transmission distance up to $30 \mathrm{~m}$ above, in the empty ore no obstacle space up to loom above, if additional CC2591 distance extension module can increased to more than $250 \mathrm{~m}$. In summary, the system design meets the design requirements, and achieves the objective of "remote and accurate control of the host computer centralized management" .

\section{ACKNOWLEDGMENT}

This work is supported by NFSC(61302179), and key science foundation for outstanding young talent of Anhui
(2013SQRL072ZD), and Anhui provincial natural science foundation(1301023004), West Anhui University research cooperation project (2014LWA001).

\section{REFERENCES}

[1] Lynn Choi, Jae Jung. M-Recast: Robust and Energy-Efficient Geometric Routing for Mobile Sensor Networks [J]. Lecture Notes in Computer Science, 2008(5): 304-316

[2] Nauvoo V, Gross R. Connectivity-Aware Routing (CAR) in Vehicular Networks. [C]. IEEE, 2007(5):1919 -1927

[3] Stemmers R, Meaning H. Reducing Energy Computation of Network Interfaces Hand Devices [C].IEICE Transactions on Communications 2007(8):125-131.

[4] Baker N. ZigBee and Bluetooth Strengths and Weaknesses for Industrial Applications [J]. Computing \& Control Engineering Journal, 2005, 16(2)20-25.

[5] Akyldiz IF, Su W, Sankarasubramanian Y, Cayirci E. Wireless sensor networks: A Survey [J].Computer Networks, Mar., 2012, 38(04): 393-422.

[6] Ye Xiaojing, Huang Junwei. A framework for Cloud-based Smart Home[C]. 2011 International Conference on Computer Science and Network Technology, 2011(2):894-897.

[7] Jhuizink J, Konjnenburg M. An Ultra-Low Energy Biomedical Signal Processing System Operating at Near-Threshold[J]. IEEE Transactions on Biomedical Circuits and System, 2011(6):546-554.

[8] Richard G, Craig A. Performance Modeling Of Daylight Integrated Photo Sensor-Controlled Lighting Systems[C]. Proceedings of the 2011 Winter Simulation Conference, 2011:903-914. 